\title{
La formación de científicos creativos con perspectiva regional en las universidades públicas: un reto
}

\section{The training of creative scientists with a regional perspective in public universities: a challenge}

\section{DOI: $\underline{10.32870 / \text { dse.v0i19.514 }}$}

María del Rosario Pineda-López*

Lázaro R. Sánchez-Velásquez**

Enrique Alarcón-Gutiérrez ${ }^{* * *}$

Edgar Eduardo Ruiz Cervantes****

\begin{abstract}
Resumen
Las universidades públicas se han convertido paulatinamente en centros de formación, desarrollo cognitivo y tecnológico, en las que se promueve una ciencia un tanto desvinculada de la sociedad y sus problemáticas. Así, surge una necesidad de construir plataformas interdisciplinarias y sistémicas. Considerar en la formación académica enfoques de creatividad colectiva y con perspectiva humanista, resultaría en un cambio de paradigmas ante la crisis socioambiental que enfrentamos, desde una escala local/regional. Se presentan algunas reflexiones de los autores a partir de su experiencia docente y de investigación en los niveles de licenciatura y posgrado, respecto a la importancia de eliminar enfoques rígidos y fomentar la creatividad e inteligencia colectiva. Se plantea la necesidad de integrar, en el seno de las universidades públicas, problemáticas regionales como elementos que den pertinencia a la investigación y generen científicos y profesionistas creativos con perspectiva local y regional.
\end{abstract}

Palabras clave: científicos - creatividad - México - sociedad - universidad.

\section{Abstract}

Public universities have gradually become centers of training, cognitive and technological development, promoting a science somewhat dissociated from society and its problems, creating the need for interdisciplinary and systemic platforms. Considering creative and collective ap-

\footnotetext{
* Doctora en desarrollo comunitario. Académica de la Universidad Veracruzana. Línea de Investigación: Biodiversidad gente y servicios ecosistémicos. Instituto de Biotecnología y Ecología Aplicada (INBIOTECA). México. rosariorosariop@gmail.com

** Doctor en biología. Académico de la Universidad Veracruzana. Línea de Investigación: Biodiversidad gente y servicios ecosistémicos. Instituto de Biotecnología y Ecología Aplicada (INBIOTECA). México. lasanchez@uv.mx

*** Doctor en ciencias. Académico de la Universidad Veracruzana. Línea de Investigación: Biodiversidad gente y servicios ecosistémicos. Instituto de Biotecnología y Ecología Aplicada (INBIOTECA). México. enalarcon@uv.mx

**** Doctor en sociología. Técnico Académico de la Universidad Veracruzana. Línea de investigación: Diálogo de saberes y sustentabilidad humana para el buen vivir. Centro de Eco-Alfabetización y Diálogo de Saberes (EcoDiálogo). México. edgruiz@uv.mx
} 
proaches to education with a humanistic perspective would lead to a paradigm shift regarding the socio-environmental crisis that we face, at a regional scale. Some reflections of the authors are presented, based on their teaching experience at the undergraduate and graduate levels, regarding the importance of eliminating rigid approaches and promoting creativity and collective intelligence. The need to integrate regional and local problems as elements that make research within universities more relevant is also discussed.

Key words: creativity - Mexico - society - scientists - university.

\section{Introducción}

Las universidades públicas se han convertido progresivamente en centros de formación, desarrollo cognitivo y tecnológico. Aunque se reconoce que en ellas se realiza gran parte de la investigación (Arocena y Sutz, 2001; Sánchez-Velásquez et al., 2010), también promueven una ciencia un tanto desvinculada de la sociedad y sus complejas problemáticas. Surge entonces la necesidad de construir plataformas interdisciplinarias y sistémicas que permitan generar conocimientos articulando enfoques ecológicos, culturales, tecnológicos, políticos (Badillo Islas, 1996) sociales y científicos, por lo que es necesario analizar cómo estamos abordando la construcción del conocimiento en las universidades públicas (Lanz et al., 2003).

Estas instituciones son espacios de reflexión y análisis sobre el tipo de vida que queremos llevar, en qué mundo queremos vivir y hacia dónde podemos dirigir la ciencia, la tecnología y las humanidades (Wilson, 2016) para lograr objetivos comunes (Tudge, 2003). Así, las universidades públicas representan plataformas para el desarrollo y la sustentabilidad de la sociedad; sin embargo, viven en un contexto de crisis generada por las políticas neoliberales que atentan cada vez más contra estas instituciones.

Hace algunas décadas, la educación superior comenzó a estar determinada por una economía mundial basada ahora en los capitales transnacionales. Esto la llevaría poco a poco a la competencia salvaje que este modelo económico ha ido imponiendo a todas las instituciones de educación superior (Ruiz, 2016). Con estas políticas, se han generado profundos desafíos de tipo político y económico que están afectando al sector educativo, especialmente — dada su historia y composición- a la educación pública superior.

Las sociedades modernas se conciben como una serie de estructuras organizacionales fragmentadas, sin una visión sistémica y compleja; de esta manera, dependiendo de cómo concibamos la sociedad, el ambiente y el desarrollo social, es la forma en que nos relacionamos con estos temas. En el nivel mundial existen dos escuelas sociológicas relacionadas con el estudio del desarrollo de la sociedad, la escuela individualista y la escuela colectivista (Padrón, 2005). Desde la perspectiva de una universidad, asumir alguna de estas corrientes significa, por lo tanto, asumir como institución una visión-posición individualista o colectivista, lo que se verá reflejado, por ejemplo, en el tipo de investigación a desarrollar (Padrón, 2005).

Diálo@os sobre Educación año 10 | número 19 | julio-diciembre 2019 | ISSN 2007-2171 
No solo se trata de trabajar en colectivo y para el colectivo de la sociedad. Desde nuestra perspectiva, es necesario asumir este enfoque, ya que los problemas socioambientales son complejos y rebasan los enfoques unidisciplinares. Es necesario transitar hacia la transdisciplinariedad (Nicolescu y Gómez, 1996) o, por lo menos, iniciar con una ciencia interdisciplinaria, más allá de un enfoque lineal y reduccionista. Las universidades no son entes independientes y desvinculados; al contrario, forman parte importante de la sociedad y son —-todavía - una de las instituciones con mayor credibilidad social.

\section{Desarrollo}

Existe la necesidad de una revaloración de los saberes, de la redistribución del conocimiento y el acceso a las tecnologías apropiadas y apropiables. En este sentido, las universidades, al estar ligadas tanto a la formación de cuadros científicos y profesionistas como a la generación del conocimiento y de tecnología, están en la posibilidad de contribuir con soluciones y productos viables a las problemáticas socioambientales complejas dentro del marco de la interacción ciencia-sociedad.

El aprendizaje se consolida desde la interacción con el entorno, el cual se da a través de un flujo bidireccional que retroalimenta el aprendizaje mediante la formación que surge a partir de las modificaciones que en dicho entorno realiza. Esta relación ofrece, además, la oportunidad de que el binomio universidad pública-entono local/regional haga que la institución en cuestión forme parte de este, reafirme su pertenencia y asuma una responsabilidad real y consciente, al mismo tiempo que se nutre de la información obtenida por su accionar (Guillaumin-Tostado, 2000). De esta manera se estaría contribuyendo a la formación de jóvenes científicos con una perspectiva local/regional.

Desde este enfoque, la extensión y vinculación — que están íntimamente relacionadas con el entorno- pueden considerarse como herramientas útiles para captar y sistematizar problemáticas, identificar la demanda de conocimientos y tecnologías, etc., que podría abordar la ciencia y diseñar programas de formación docente, con lo que se generarían nuevos conocimientos y tecnologías, que a su vez representarían insumos para una nueva manera de ser de la universidad. Con esto se estarían ofreciendo productos creados por la propia formación universitaria, en donde la docencia, vinculación e investigación se articulan desde una nueva manera, integrando sistémicamente conocimientos enfocados hacia el desarrollo local y regional.

Así, se promueve una nueva forma de construir conocimiento entramado e interconectado, centrado en la formación de jóvenes profesionistas creadores de futuros sostenibles y con vocación de servicio. Sin embargo, el modelo - aún napoleónico y fragmentado de las universidades - se limita a enseñar e investigar lo que se hace en los países industrializados cuya realidad, presupuesto y entorno no son equiparables con los países en desarrollo; incluso, en ocasiones, ni entre los propios de otros países de Latinoamérica. Es decir, las agendas de la ense- 
ñanza y la investigación surgen principalmente desde los países desarrollados, ya sea mediante las políticas del Banco Mundial, de nuestros paisanos egresados y formados en esos países, u otros procesos ligados a la información.

No obstante, en México hay iniciativas en las que se promueve el desarrollo de una investigación dirigida a la solución de problemas locales/regionales con la finalidad de generar profesionistas y científicos creativos conscientes de su entorno. A continuación, mencionaremos brevemente algunas experiencias que esperamos puedan ser útiles para otras universidades del país.

La Universidad de Guadalajara ha puesto en práctica en la Costa Sur del estado de Jalisco, a través del Departamento de Ecología y Recursos Naturales, un modelo que integra la conservación en la Reserva de la Biosfera Sierra de Manantlán (RBSM). Consiste en un programa de formación de estudiantes (ingenieros en Recursos Naturales y Agropecuarios) y una entidad académica universitaria cuyas líneas de investigación están dirigidas, no solo a la conservación de la RBSM sino, también, a las necesidades y demandas regionales en las que se integra a su vez a los estudiantes (Martínez, et al., 2006).

Otro ejemplo, aunque con un enfoque diferente, es la Universidad de la Tierra en Oaxaca, en donde la formación es un aspecto de la vida cotidiana y el plan de estudios es un ejercicio autónomo que gira alrededor de problemáticas locales. Las investigaciones teóricas y aplicadas están relacionadas con algún aspecto de la realidad local o regional, siendo estos verdaderos ejercicios de reflexión en la acción (Universidad de la Tierra en Oaxaca, 2009).

En la Universidad Veracruzana, al menos existen dos centros de investigación que han intentado integrar el enfoque regional a su esquema de trabajo en la docencia y la investigación, ambos ubicados en la capital del estado. Por un lado, el Centro de Eco-Alfabetización y Diálogo de Saberes de la Universidad Veracruzana, fundado en el año 2006, el cual ha generado Líneas de Generación y Aplicación del Conocimiento (LGAC), así como distintos programas de formación: seminarios, diplomados y posgrados, con una perspectiva creativa, transdisciplinaria y con un enfoque de diálogo de saberes. Esta herramienta —según la experiencia de los profesorespermite acercar la distancia que convencionalmente existe entre la ciencia y sus componentes. Y, por otro lado, el Instituto de Biotecnología y Ecología Aplicada (INBIOTECA), fundado en 2004, cuya perspectiva está intentando lentamente desarrollar investigaciones que integren herramientas y enfoques metodológicos tanto de la biotecnología como de la ecología aplicada, dirigidas a responder a las necesidades locales/regionales en el sector agrícola y forestal con programas formativos integrados en dos posgrados (PNPC por Conacyt) y tres cuerpos académicos consolidados.

Siguiendo con nuestro planteamiento, es necesario subrayar que el entorno de las universidades está constituido por una amplia gama de factores diversos, complejos e interdependientes, tales como los ambientales, económicos, sociales o culturales, entre otros, por lo que, dependien- 
do del tipo de investigación que se desarrolle, deberían ser considerados como parte del contexto de esta. Así, en términos de procesos, dinámicas y energía, de acuerdo con Bohm y Peat (1988), todo se encuentra relacionado; lo que algunos físicos han llamado el paradigma holográfico. Estos autores señalan que la ciencia es un intento de entender el universo y la relación del hombre con la naturaleza. ¿Cómo es posible entonces que, a partir de este enfoque, se dé como resultado la fragmentación del conocimiento en disciplinas como la física, la química y la biología, etc., y cada una de estas en especializaciones? (Guevara, 1991).

Las universidades siguen siendo espacios que albergan una alta proporción de especialistas en disciplinas predeterminadas —en su gran mayoría creadas artificialmente- y que contrastan con una problemática social. Se necesitan instituciones e individuos que tengan una visión más amplia, que rebase los límites de las disciplinas o que se suban, desde su disciplina, a una plataforma más compleja y sistémica, que puedan ser capaces de enfocar a profundidad los problemas que enfrenta la sociedad y su ambiente. En un contexto regional, debemos continuar con la reflexión acerca de cómo construir y consolidar el espacio iberoamericano de conocimiento, basado en una propuesta de educación en ciencia, tecnología y sociedad para la educación superior, con énfasis en la participación pública (Osorio y Martins, 2010).

Cada vez más se percibe que los conocimientos encasillados en disciplinas no resuelven los problemas complejos; estos son multidisciplinarios, multidimensionales, transversales, globales, transdisciplinares; pensemos tan solo en un tema: el cambio climático global. Edgar Morin (2010) señala que "la hiper-especialización impide ver lo global y lo esencial, los problemas esenciales nunca son parcelarios, y los problemas globales son cada vez más esenciales [...] fraccionar la complejidad de los problemas, disminuye las posibilidades de comprensión (Morin, 2000). Por lo que, citando a Gedeón-Zerpa y García-Yamín (2009), “Las universidades concebidas como centros de difusión del saber científico, deben visionarse desde la Transdisciplinariedad con miras a la formación holística de profesionales con competencias que les permitan abordar la compleja realidad social en la que se desenvuelven".

¿Por qué se da la fragmentación?, ¿se debe a algún error en el enfoque científico, en los métodos utilizados o en los objetos de estudio? Tal fragmentación tiene su origen en la manera en que los seres humanos perciben y actúan (Bohm y Peat, 1988), y que desde la perspectiva científica se ha denominado infraestructura tácita de las ideas científicas. Esta división de un determinado campo de la ciencia condujo a lo que conocemos como la hiperespecialización (Gedeón-Zerpa y García-Yamín, 2009). Según Bohm (1997) “las habilidades tácitas del científico lo llevan a avanzar en su investigación utilizando técnicas experimentales específicas, sin tomarse el tiempo muchas veces, de cuestionarse sobre los detalles de lo que se está haciendo y su contexto". De tal suerte que, al estar "acostumbrados" a utilizar sus habilidades y conocimientos de manera subliminal e inconsciente, existe una tendencia mental de aferrarse a ellos, a lo que es familiar, a lo que sabe hacer, a seguir en su "zona de confort" (Bohm, 1997). 
Además, esta tendencia es a su vez reforzada por las instituciones de las que dependen la seguridad profesional del científico (Kuhn, 2011) y "heredada" a los estudiantes. Interpretando a Kuhn (2011), los científicos que lideran algún paradigma de la ciencia heredan a sus discípulos, no solo conocimientos, sino también comportamientos, es decir, si el líder es ególatra o humilde, existen grandes probabilidades que sus discípulos sean ególatras o humildes, según el caso. De esta manera, los discípulos o seguidores de un paradigma también son producto de lo que Morin (1999) llama imprinting cultural, el cual es influenciado, trasmitido, consciente o inconscientemente por los profesores a los futuros profesionistas e investigadores.

¿Qué podemos hacer para modificar esta tendencia? Proponemos atrevernos a romper y reconstruir paradigmas, actitudes y comportamientos; entrenar a nuevos jóvenes científicos fomentando su creatividad e inteligencia colectiva, la cual descansa en la habilidad de tener nuevas percepciones en las que la comunicación y el diálogo son fundamentales; fomentar espacios de reflexión sin etiquetas, prejuicios y "suspendiendo" nuestro imprinting cultural y disciplinar, acercándolos a realidades desde un enfoque multidisciplinar y planteando preguntas de investigación desde la complejidad de las problemáticas.

Al abordar problemas desde su complejidad y su posible solución desde la colectividad, surge la creatividad e inteligencia colectiva. Citando a Bohm y Peat (1988), "Vivir de manera creativa requiere una percepción extremadamente sensible de los órdenes y estructuras de relación de los individuos, la sociedad y la naturaleza". Es entonces importante considerar que las universidades —en términos de la enseñanza-aprendizaje-, impulsen y fortalezcan en los estudiantes la aptitud de conscientizar, de ver los problemas de manera compleja e integral —con miras a la transdisciplina-, despertando en ellos la curiosidad y la creatividad. En un sentido más amplio, Wilson (2016) plantea la unificación de conocimientos integrando las ciencias con las humanidades, lo que él llama la Segunda llustración.

¿Por qué es importante fomentar la creatividad en la formación universitaria? La historia de la ciencia ha demostrado que muchas de las principales aportaciones provienen de científicos jóvenes (Guevara, 1991), por lo que la universidad se constituye en el momento y el espacio adecuado para promover el potencial creativo innato de los estudiantes.

No debemos perder de vista que, en la actual época de globalización, el conocimiento se ha convertido en la materia prima de mayor valor y de mayor movilización, generando una nueva economía de la educación asociada a derechos de autor, a la creación de patentes, a su comercialización, a los acuerdos empresariales globales, al pago a los académicos por su productividad (Rama, 2006; 2007). Todo esto está generando una transformación no solo de los modos de producción y de transmisión de los conocimientos, sino también de los paradigmas epistemológicos sobre los cuales se estructuran y desarrollan las universidades (Rama, 2006; 2007), lo que se conoce como capitalismo académico (González, 2000). 
Es un reto incluir en el proceso de la investigación y formación académica, la solidaridad, la honestidad, la humildad y la otredad, entre otros valores, en el contexto de una creatividad e inteligencia colectiva. Por ejemplo, en el colectivo de una clase, los estudiantes tienen mucho que aportar mediante la reflexión, la crítica y la autocrítica, el cuestionamiento, la praxis y el diálogo, este último, como Bohm (1997) lo sugiere, sin prejuicios y sin asunciones. Recordemos que muchos de esos estudiantes serán tomadores de decisión en la academia, en las empresas, en los gobiernos, en las instituciones y en casa.

A lo largo de la historia humana han surgido importantes civilizaciones que representaron un parteaguas para la sociedad contemporánea. Perutz (2002) se pregunta ¿cómo logró la Florencia del siglo XV, con una población de apenas 50 mil habitantes, concebir al mismo tiempo grandes artistas como Leonardo Da Vinci, Miguel Ángel, Rafael, Brunneleschi, Alberti, Ghiberti, entre otros? o ¿Cómo logró la Francia del siglo XIX producir un Renoir, Cézanne, Degas, Monet, Manet, Toulouse-Lautrec o Seurat?Y se responde: gracias a la creatividad (Perutz, 2002); a lo que Albert Einstein llamaba la inteligencia divirtiéndose. ¿Cómo se divierte la inteligencia? MacKinnon (1963) decía que "hay que jugar con el problema más tiempo antes de intentar resolverlo", lo cual requiere controlar la ansiedad y ser más tolerantes.

De acuerdo con Arnold Toynbe (citado por Bohm y Peat, 1988), existe un ciclo en la aparición de nuevas sociedades, las nuevas civilizaciones se originan a partir de una oleada creativa que quizá se exprese fuertemente en algunos individuos como resultado del ambiente histórico, social y condiciones ambientales en general, y poco a poco va permeando al conjunto de la sociedad. Dicho autor señala que a partir de esta oleada las civilizaciones llegan a florecer, se mantienen durante un tiempo y en algún momento colapsan. Por ejemplo, el Renacimiento, donde descansan las raíces de la civilización actual, representó quizá la época más creativa - para bien o para mal - en la que aparecieron propuestas de un nuevo orden, tanto en las artes como en las ciencias.

Hoy, a nuestra civilización se le da una temporalidad menor a los mil años porque ya no sigue el ciclo continuo de las civilizaciones, no somos una sociedad creativa (Perutz, 2002). Nos estamos hiperespecializando de manera sistemática y tecnológica, encajonándonos, sin considerar a la creatividad como algo necesario. Hoy estamos enfrentando una etapa de aniquilación a escala planetaria en diversos órdenes: ambientales, económicos y sociales (Bohm y Peat, 1988). Frente a esto, es momento de reconocer que ya no es posible continuar con enfoques rígidos y reduccionistas en la generación del conocimiento; cada vez es más necesaria la interacción entre disciplinas para abordar problemas que, como ya hemos dicho, son multifactoriales.

En México, la propia estructura de las universidades, así como los sistemas del gobierno federal como el Consejo Nacional de Ciencia y Tecnología, en lo general, y el Sistema Nacional de Investigadores (SNI) en lo particular, vinculados directamente al fortalecimiento de la ciencia, parcelan las investigaciones en disciplinas, y con ello limitan la interdisciplina y mucho más la 
transdisciplina. El enfoque de estos sistemas gubernamentales disminuye las oportunidades para la interacción y creación de líneas de trabajo que integren enfoques holísticos que den respuestas más integrales, co-inteligentes y creativas. Vale la pena mencionar que la estructura de pensamiento científico, generada por el SNI, ha girado hacia la producción de artículos como el fin, y no como el medio, para abordar los grandes problemas sociales y ambientales que aquejan al país.

La interdisciplinariedad — debemos recordar — es la base fundamental para la resolución de problemas de manera conjunta, con creatividad e inteligencia colectiva, y se la ha denominado también proceso de convergencia; es decir, representa el proceso de comunicación y colaboración entre distintas disciplinas (Amaro y Robles, 2013). Ante esto, caben las preguntas siguientes: ¿En qué medida las y los investigadores estamos dispuestos a abordar, desde la complejidad, diversas problemáticas, integrando, interactuando y trascendiendo más allá de nuestras disciplinas? ¿De qué forma nuestros egos disciplinarios e individuales lo permitirán? ¿Qué tan dispuestos estamos para suspender nuestras creencias, prejuicios y etiquetas, y dialogar profundamente entre disciplinas?

En febrero de 2012, en México, instituciones como la Universidad Nacional Autónoma de México, el Instituto Politécnico Nacional, el Centro de Investigaciones y Estudios Avanzados, el Consejo Nacional de Ciencia y Tecnología, la Red Nacional de Ciencia y Tecnología, entre otras, propusieron la Agenda Ciudadana de Ciencia, Tecnología e Innovación (Franco et al., 2013). Esta agenda inició con una consulta nacional realizada por primera vez en el país, en la que se puso a consideración de la sociedad una serie de temas prioritarios para darles atención. Algunos de los objetivos eran: establecer canales de comunicación con la sociedad y traducir la opinión ciudadana en un mandato sobre la agenda en ciencia y tecnología.

Todo esto frente a un horizonte de 30 años, y en un contexto no solo nacional sino dentro de una agenda iberoamericana de ciencia y tecnología, en la que México es el primer país en tomar la estafeta. En esta encuesta nacional se obtuvieron 300 mil repuestas y arrojó los primeros tres temas prioritarios que la sociedad identifica como urgentes. 17 \% identificó el tema de la educación (modernizar el sistema educativo con enfoque humanista, científico y tecnológico), $15 \%$ señaló el tema del agua (asegurar el abasto de agua potable para toda la población), y en tercer lugar, $13 \%$ consideró prioritario recuperar y conservar el ambiente para mejorar nuestra calidad de vida.

Si bien el porcentaje de la población que participó fue bajo, el ejercicio puso de manifiesto la preocupación que parte de la sociedad tiene respecto a la formación de recursos humanos desde una perspectiva, no solo científica y tecnológica, sino humanista. Con ello, debemos tener presente que en las universidades se trasmite a los estudiantes no solo conocimientos, sino también valores humanos como la honestidad, la virtud, el respeto, la nobleza, la solidaridad y la humildad, entre otros, que deben distinguir a una sociedad o un país y, por lo tanto, valores 
que deberían estar fuertemente involucrados en los enfoques que demos a la investigación y a la formación de recursos humanos.

Necesitamos marcos epistémicos transversales cuyo objetivo sea la generación de opciones humanas y sustentables capaces de contender con la compleja y generalizada crisis que hoy en día vivimos. Citando a Wilson (2016): "El estudio de la relación entre la ciencia y las humanidades debería estar en el centro de la educación liberal [...] ya sean facultades de ciencias o de humanidades.

\section{Consideraciones finales}

El presente ensayo se limita únicamente a compartir una reflexión colectiva desde nuestras diversas experiencias, primero como estudiantes, y hoy como docentes/investigadores en universidades públicas mexicanas. Parte de las experiencias se han dado en aulas, laboratorios, comunidades rurales, áreas naturales protegidas, bosques, agro-ecosistemas, etc. a través de la interacción con otros investigadores, algunos de ellos fueron nuestros estudiantes y hoy son colegas, nos empujaron a abrirnos a compartir estas reflexiones con todas las limitaciones que se puedan identificar.

Para nosotros, la creatividad está inherentemente vinculada a la ciencia. Si partimos del hecho que la creatividad es una propiedad inherente al funcionamiento del cerebro humano (Guevara,1991), y que el desarrollo histórico de la ciencia ha hecho partícipes de manera significativa a los jóvenes, es entonces propicio considerar que en esta etapa de juventud es necesario fomentar la creatividad en la ciencia, en lo individual y en lo colectivo.

Fomentar la creatividad e inteligencia colectiva en los jóvenes científicos, como instrumento de cambio y apertura, teniendo en mente que la investigación puede surgir del proceso bidireccional entorno-investigación-entorno, permitirá establecer una importante diferencia pues con esta visión se estará al servicio de la sociedad, que está esperando respuestas, no solo de calidad académica sino, también, que aporten en la resolución de la crisis socioambiental en la que vivimos.

Una propuesta que nos permitimos hacer, es conjuntar integralmente los esfuerzos en las universidades públicas enlazando las Líneas de Generación y Aplicación del Conocimiento enfocadas a problemáticas regionales y/o locales ligadas a la experiencia docente. Esto requeriría de la correspondencia en la estructura y políticas institucionales internas de cada universidad que lo faciliten, favoreciendo así la formación de jóvenes científicos en las regiones donde se asienten las universidades públicas.

La transformación en las universidades públicas es más compleja que una reforma organizacional, tecnológica o estructural. Está íntimamente relacionada con la integración de una perspectiva local/regional de cada institución universitaria, integrando la visión, el sentir, las experiencias de quienes hacen ciencia y viven en esas regiones. 


\section{Referencias}

Amaro, M. y E. Robles (2013). "Producción de conocimiento científico y patrones de colaboración en la biotecnología mexicana". Entreciencias: Diálogos en la Sociedad del Conocimiento, 1(2), 183-195. www.entreciencias.enes.unam.mx

Arocena, R. y J. Sutz (2001). “La universidad latinoamericana del futuro”. En G. Rodríguez-Ortiz y B. Pérez-González (eds.). Tendencias-escenarios. México: Editorial UDUAL. https://www.oei. es/historico/salactsi/sutzarocena01.htm

Badillo-Islas, L. F. (1996). "Las perspectivas del conocimiento científico-técnico en la sociedad contemporánea". Economía. Teoría y Práctica, Nueva Época, 6. http://biblioteca.clacso.edu. ar/gsdl/cgi-bin/library.cgi?c=mx/mx-022\&a=d\&d=HASH013769be8d2cec6903df6c66

Bohm, D. (1997). Sobre el diálogo. Barcelona: Editorial Kairós.

y F. D. Peat (1988). Ciencia, orden y creatividad: las raíces creativas de la ciencia y la vida. Barcelona: Editorial Kairós.

Franco, J., R. López, R. Ruiz, F. Castellón, A. Tello, R. Asomoza y J. Narro (2013). “Informe público de la Agenda Ciudadana de Ciencia, Tecnología e Innovación". http://www.agendaciudadana. mx/index.php

Gedeón-Zerpa, I. y N. García-Yamín (2009). “La transdisciplinariedad en la educación superior del siglo XXI". Revista de Artes y Humanidades UNICA, 10(3): 58-70.

Guevara-Pozas, Á. D. (1991). "Creatividad y ciencia". Revista Mexicana de Ciencias Políticas y Sociales, 36(144), 81-90. México: UNAM.

Guillaumín-Tostado, A. (2001). “Complejidad, transdisciplina y redes hacia la construcción colectiva de una nueva universidad". Polis en línea. http://journals.openedition.org/polis/8085

González, C. P. (2000). "La nueva universidad". http://www.ceiich.unam.mx/educacion/casanova.html

Kuhn, T. S. (2011). La estructura de las revoluciones científicas. México: Fondo de Cultura Económica.

Lanz, R., A. Fergusson y A. Marcuzzi (2016). "Procesos de reforma de la educación superior en América Latina". En E. Rodríguez-Zidan. Revista Educación y Ciencias, 5(46), 45-55. México: Universidad Autónoma de Yucatán.

MacKinnon, D. W. (1963). "The Identification of Creativity". Applied Psychology, 12(1), 25-46.

Martínez, L. M., P. R. W. Gerritsen, R. Cuevas y J. A. Rosales (2006). "Incorporating Principles of Sustainable Development in Research and Education in Western Mexico". Journal of Cleaner Production, 14(9), 1003-1009.

Morin, E. (1999). Los siete saberes necesarios para la educación del futuro. París: Unesco. (2000). La mente bien ordenada: repensar la reforma, reformar el pensamiento. Barcelona: Seix Barral.

Nicolescu, B. y M. V. Gómez (1996). La transdisciplinariedad. Manifiesto. París: Multiversidad Mundo Real Edgar Morin, AC. 
Osorio C. y I. P. Martins (2010). "La educación científica y tecnológica para el Espacio lberoamericano de Conocimiento". En M. Alborns y J. A. López Cerezo (eds.). Ciencia, tecnología y universidad en Iberoamérica. Buenos Aires: Eudeba.

Padrón, G. J. (2005). “Investigación, universidad y sociedad”. Conferencia. II Seminario Internacional EDUCA. Instituto Pedagógico Rural Gervasio Rubio. Universidad Pedagógica Experimental Libertador, Venezuela.

Perutz, M. F. (2002). Los científicos, la ciencia y la humanidad: ojalá te hubiese hecho enojar antes. Barcelona: Ediciones Juan Granica. (2007). Los postgrados en América Latina en la sociedad del conocimiento. México: Fondo Editorial Ipasme.

Rama, C. (2006). "La tercera reforma de la educación superior en América Latina y el Caribe: masificación, regulaciones e internacionalización". Revista Educación y Pedagogía, 18(46). Colombia: Universidad de Antioquia, 11-24.

(2007). Los postgrados en América Latina en la sociedad del conocimiento. Caracas: Fondo Editorial Ipasme.

Rama, C. (2006). "La tercera reforma de la educación superior en América Latina y el Caribe: masificación, regulaciones e internacionalización". Revista Educación y Pedagogía, 18(46), $11-24$.

Ruiz, E. (2016). "La universidad en crisis, la posibilidad del diálogo". En A. Domingo y P. Francesco (eds.). Reaprendizaje transdisciplinario. México: Colección BIBLIOTECA, 173-190.

Sánchez-Velásquez, L. R., M. R. Pineda-López, P. Gerez-Fernández y R. Lara-González (2010). "Ciencia, universidades públicas y su contribución al conocimiento de la biodiversidad". Conocimiento-Cultura-Ciencia, 2(4), 13-18.

Tudge, C. (2003). “Clonación: ¿quién tiene derecho a hacer qué y a quién?". Clonación. Madrid: Universidad Complutense, 17-40.

Universidad de la Tierra en Oaxaca (2009). ¿Qué es Unitierra? http://unitierra.blogspot.mx/ Wilson, E. (2016). El sentido de la existencia humana. España: Gedisa. 